\title{
Single-molecule techniques and cell-free protein synthesis: a perfect marriage
}

\author{
Alexandros Katranidis ${ }^{1}$ and Jörg Fitter ${ }^{1,2}$ \\ ${ }^{1}$ Forschungszentrum Jülich, Institute of Complex Systems ICS-5, Jülich, Germany \\ ${ }^{2}$ RWTH Aachen, I. Physikalisches Institut (IA), Aachen, Germany \\ Correspondence should be addressed to a.katranidis@fz-juelich.de Tel.: +49 24616185474 Fax: +49 2461611448 \\ or fitter@physik.rwth-aachen.de Tel.: +49 2418027209 Fax: +49 2418022331
}

\begin{abstract}
Single-molecule techniques are nowadays an essential tool to study conformational changes as well as synthesis and folding of proteins. However, preparation of suitable protein samples is often time consuming and demanding. The rapid development of cell-free protein synthesis over the last years opened new perspectives for fast and easy sample preparation but this was not fully exploited until now. Here, we take a look at the advancements in sample preparation as well as in the development of technical approaches and analytical tools, which unavoidably lead to the combination of single-molecule techniques and cell-free protein synthesis. It's an ideal combination that can unlock the full potential of studying complex biological processes in the near future.
\end{abstract}




\section{Introduction}

Single-molecule techniques have emerged in recent years as a powerful tool allowing the realtime observation of complex biological processes ${ }^{1-6}$. Most of these processes proceed asynchronously or exhibit heterogeneities. In contrast to ensemble measurements, singlemolecule measurements do not need a synchronization of molecular events and can also provide detailed information on coexisting subpopulations.

The widely used single-molecule fluorescence techniques and in particular single-molecule Förster resonance energy transfer (smFRET) are ideal for studying protein structural and conformational dynamics ${ }^{1,2,5}$ or protein folding ${ }^{3,7,8}$. On the other hand, sample preparation is very often a time consuming and laborious process, especially for smFRET measurements that require double-labeled probes (Fig. 1). Over the last years the development of cell-free protein synthesis (CFPS), in which cell integrity is not required to express proteins has opened novel paths to fast and easy protein production ${ }^{9-13}$. Typically two basic types of CFPS systems are commonly used for full-length protein synthesis: (i) optimized cell extracts, also termed lysatebased systems and (ii) the more recently developed purified and reconstituted in vitro systems (e.g., the PURE system), which employ a mixture of a minimal set of purified components ${ }^{13}$.

The absence of cell walls offers large flexibility since it allows direct manipulation of these systems, including introduction of exogenous modified components ${ }^{14-17}$. In the past, for example, optical tweezers took advantage of CFPS to emerge as a powerful force-based singlemolecule technique able to study co-translational protein folding ${ }^{18,19}$ or fluorescence-based single-molecule techniques provided information on the ribosome function ${ }^{20-22}$. Furthermore, ribosome display technologies, which are based on the formation of an mRNA/ribosome/polypeptide ternary complex were realized in CFPS systems and provide a physical linkage between phenotype and genotype ${ }^{23}$. This link between genotype and phenotype allows for the selection of polypeptides that exhibit specific properties, the latter for example generated by directed evolution. Since CFPS systems also allow for labeling of the individual components, single-molecule methods are well suited to study these systems ${ }^{24,25}$.

Hence, CFPS assays were already used routinely for many years for single-molecule studies aiming to elucidate gene expression, protein synthesis, and related aspects (Fig. 1). 
Nevertheless until now, smFRET studies in general did not profit from CFPS, largely due to the resulting low protein yields, for which partly the low incorporation efficiency of unnatural amino acids as part of this method is responsible. However, the enormous sensitivity and the practically small sample amounts needed for single-molecule fluorescence techniques suggest that CFPS and single-molecule techniques could be a perfect couple.

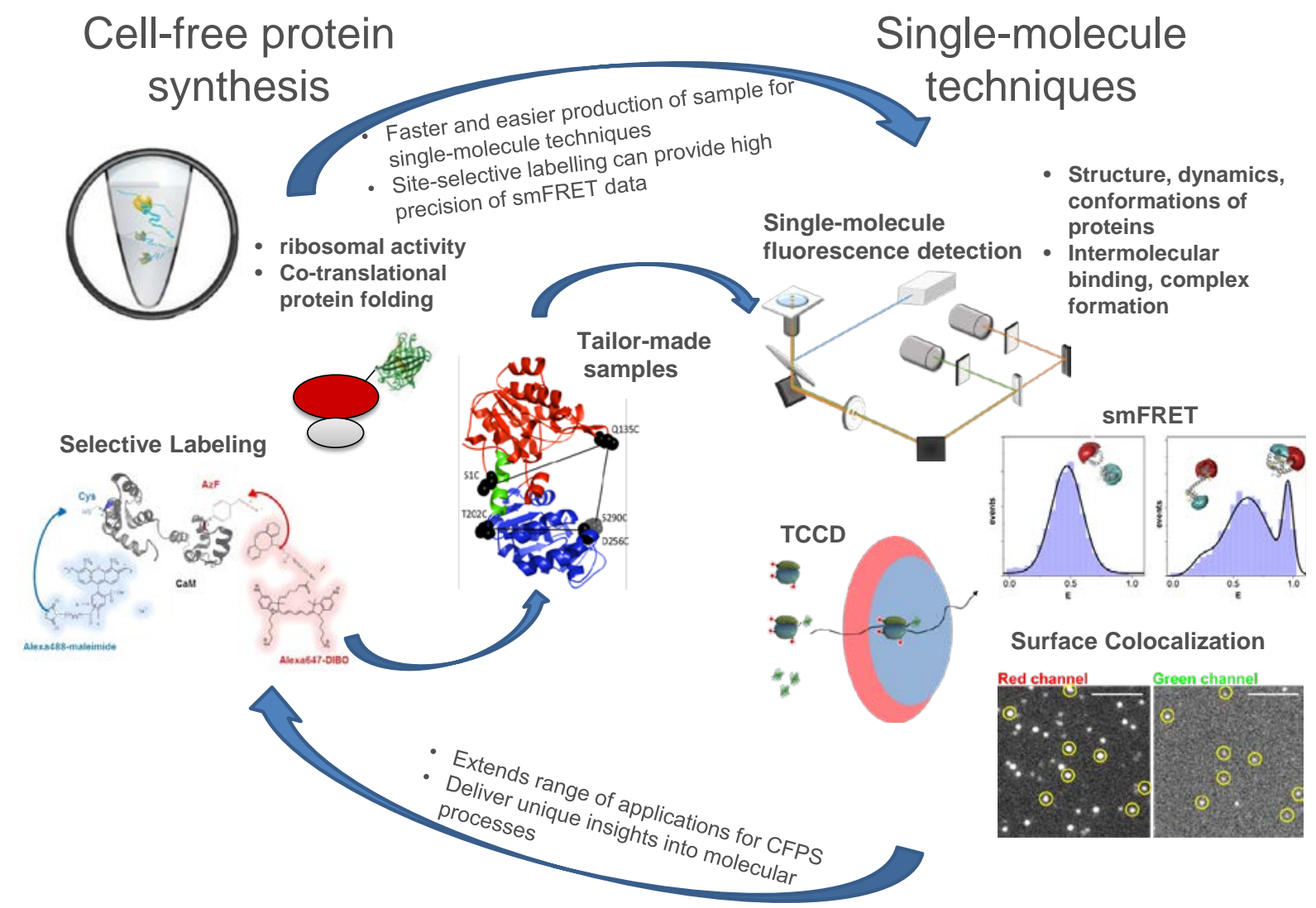

Figure 1: Complementarity of cell-free protein synthesis and single-molecule techniques. On the one hand, cell-free protein synthesis (CFPS) is ideal for producing appropriate samples for studying protein structures and conformational dynamics, as well as protein synthesis and folding with single-molecule techniques. In particular the production of proteins characterized by many different inter-dye distance pairs can benefit from the CFPS approach. Besides smFRET, also surface co-localization and two-color coincidence detection (TCCD) are employed to monitor intermolecular binding or macromolecular complex formation ${ }^{26}$. On the other hand, single-molecule studies make use of standard CFPS assays extending the range of their applications. In this respect single-molecule techniques represent unique tools to study the performance of ribosome activity or deliver molecular details about the cotranslational folding of nascent polypeptide chains. Parts of this figure adapted from ref. ${ }^{27}$. Copyright 2017 American Chemical Society and with permission from ref. ${ }^{26}$ under the terms of the Creative Commons Attribution 4.0 International License. Copyright 2017 Springer Nature. 
In this perspective we aim to recapitulate the efforts made in the field that can eventually improve the throughput in sample preparation and the characterization. From one side, new strategies were introduced that led to faster and easier production of protein samples suitable for single-molecule experiments. From the other side advanced technical approaches and analytical methods were developed that allow a better characterization of the low amounts of sample usually needed for single-molecule measurements.

\section{Cell-free production of suitable protein samples for accurate single-molecule FRET studies}

A prerequisite for smFRET measurements is the double labeling of the protein sample with two fluorescent dyes. Using the naturally occurring functional groups of a protein's amino acids practically limits site-specific labeling to a pair of cysteine residues, which can be removed or introduced at desired positions. However, due to the same functional group the two cysteine residues are randomly labeled in a non-selective manner. This can give rise to heterogeneously labeled populations that possibly make the analysis of the smFRET data difficult and ambiguous. Therefore, the last two decades considerable interest was given in alternative strategies for site-specific as well as selective protein labeling (see Fig. 2).

Evolving an orthogonal tyrosyl-tRNA/tyrosyl-tRNA synthetase pair (tRNA ${ }^{\mathrm{Tyr}} / \mathrm{TyrRS}$ ) derived from the archaeal bacterium Methanococcus jannaschii Schultz and coworkers managed to incorporate in vivo unnatural amino acids (UAAs) into proteins ${ }^{28}$. The incorporation was carried out by translating the otherwise non-sense amber (TAG) stop codon as a normal sense codon. Later, the other two stop codons, ochre (TAA) and opal (TGA) were also successfully used ${ }^{29,30}$. Several UAAs were shown to introduce a variety of novel functional groups into proteins, including ketone, azide and alkyne, which can be post-translationally labeled with fluorescent dyes in a selective manner due to their unique reactivity ${ }^{31}$. A number of smFRET studies verified the successful double labeling of proteins using the ketone - hydrazide or alkoxyamine reaction $^{32}$ and the more popular copper-catalyzed azide - alkyne cycloaddition (CUAAC) ${ }^{30,33}$. 


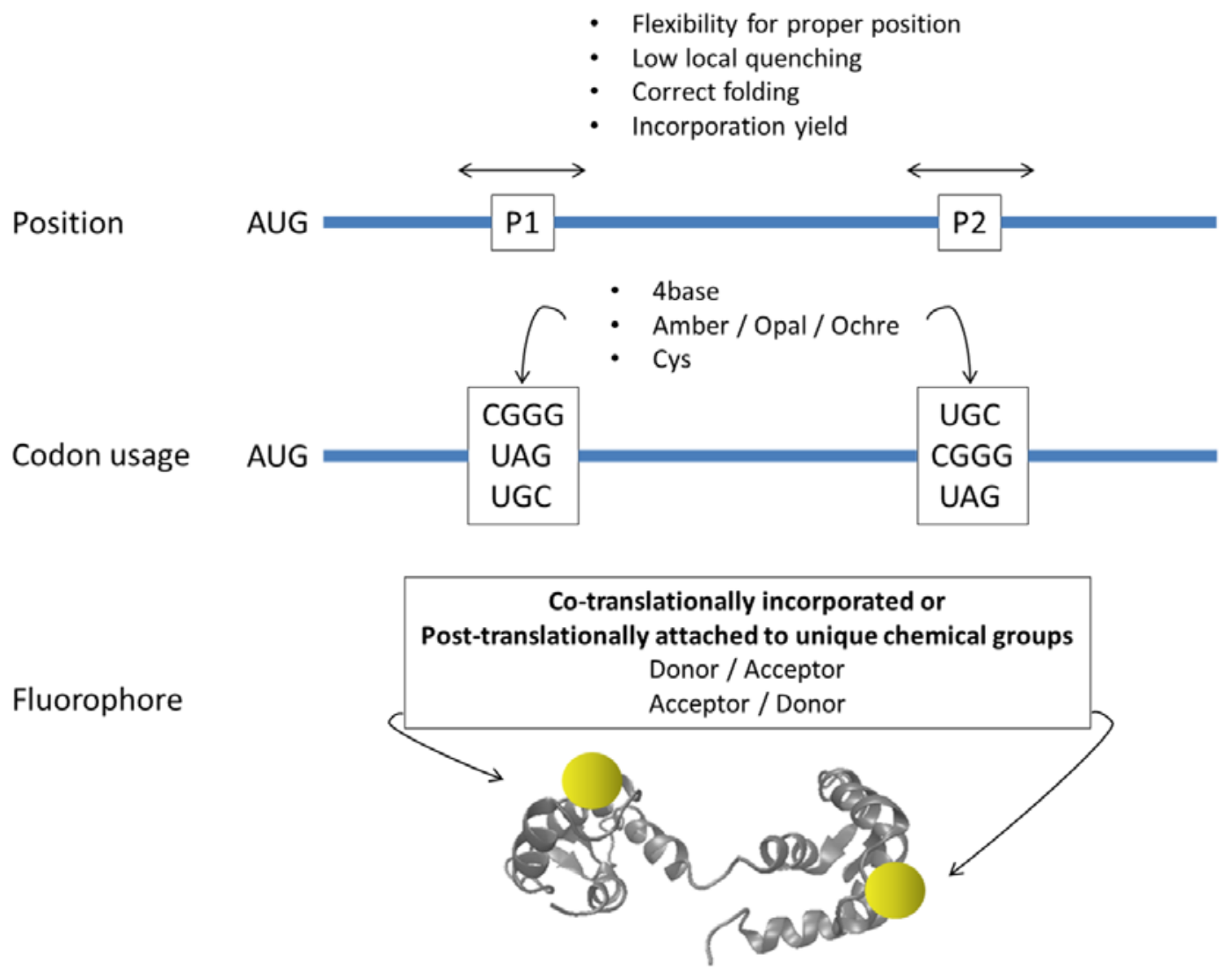

Figure 2. Levels of fine-tuning the selective labeling in the case of cell-free synthesized proteins. The exact positions of the two labels on the nascent chain can affect the incorporation yield of the UAAs. Local quenching of the fluorophores as well as hindering of the correct protein folding should be avoided. Additionally in each of the two positions, a variety of codons exhibiting different efficiencies of UAA incorporation can be used. The optimal combination of codons can be crucial. Finally, the FRET dye pair can be either incorporated directly during the synthesis of the protein or post-translationally attached to UAAs carrying unique chemical groups. Both have their advantages and disadvantages and the choice depends mostly on the applications.

However, this form of click chemistry is not optimal for biological samples due to toxicity caused by the copper-mediated generation of reactive oxygen species from $\mathrm{O}_{2}$. One way to go around it is to perform the CuAAC under anaerobic conditions $\left(<10 \mathrm{ppm} \mathrm{O}_{2}\right)^{25}$. In addition, further strategies were developed to increase the biocompatibility of the CuAAC reaction. The use of the water-soluble tris(triazolylmethyl)amine-based ligands for $\mathrm{Cu}(\mathrm{I})$ accelerate the reaction and also act as sacrificial reductants, protecting from reactive oxygen species ${ }^{34}$. Also using picolyl-azide dyes, which possess an internal copper chelating moiety, requires much lower copper concentrations lowering significantly the toxicity of the CuAAC reaction ${ }^{35}$. 
In the meanwhile, also a copper-free click reaction that utilizes ring-strained alkynes was evolved $^{36}$. Incorporation of UAAs making use of the strained-promoted azide - alkyne cycloaddition $(\text { SPAAC) })^{37}$ and also catalyst-free double labeling of proteins for smFRET measurements ${ }^{29}$ were already demonstrated. Even though, the in vivo incorporation of UAAs into proteins was a big step forward, since it allows a selective double labeling with a variety of fluorescent dyes suitable for smFRET, the whole procedure including cell-based protein synthesis, purification and labeling is rather time consuming and laborious.

Cell-free synthesis has certain advantages compared to cell-based methods, such as the rapid and exclusive production of a single protein product, as well as the active monitoring and direct manipulation of the process ${ }^{12}$. Already the co-translational incorporation of various fluorescent amino acids into proteins using CFPS was demonstrated by Sisido and coworkers ${ }^{38-}$ ${ }^{40}$. Over the years an increased number of UAAs were employed in CFPS ${ }^{41}$, including the small BODIPY dyes that were used to double label proteins selectively $y^{42,43}$. However, all reported fluorescent amino acids and the BODIPY dyes have poor photo-physical properties and weak photo-stability ${ }^{44,45}$ and are not well suited for single-molecule measurements. The next logical challenge was to combine and exploit the advantages of each of the two methods.

In a recent work from our group we were able to incorporate in a protein the chemoselective reactive $\mathrm{p}$-azido-L-phenylalanine (AzF) using CFPS ${ }^{27,46}$. This UAA introduced to the protein a unique functional group (i.e. azide), which in combination with a single cysteine residue allowed the selective double labeling of the protein with fluorescent dyes suitable for single-molecule measurements. We subsequently demonstrated that such a CFPS-based strategy is perfectly suited for performing smFRET studies. The method is limited to proteins with no or very few cysteine residues that can be easily mutated away and also still employs a C-terminal affinity purification to discard truncated products of unsuccessful UAA incorporation. Using a similar approach Hamadani and co-workers described recently the production of three dual-labeled model proteins, which were structurally characterized by SMFRET ${ }^{25}$. This latter approach has the advantage to be purification-free, but the UAA incorporation is residue-specific leading to a non-selective double labeling of the protein. 
A cell-free approach is easier to handle than the in vivo method since it requires neither cell cultures nor safety regulations for recombinant organisms, making it the method of choice for laboratories that conduct single-molecule experiments, but otherwise lack the biological facilities. The purification is also more straightforward since the protein doesn't have to be purified from all the other cytosolic proteins. In addition the cell-free approach expands the range of proteins that can be used in single-molecule studies to include difficult-to-express or toxic proteins, since synthesis takes place outside living cells.

Even though the field of protein folding has seen great progress over the past decades, more recently the focus has been placed on understanding cotranslational protein folding ${ }^{47}$. There is an increasing tendency to follow it in real time and single-molecule techniques such as smFRET are good candidates for providing direct information ${ }^{48}$. The incorporation of the fluorescent BODIPY dyes demonstrated by Sisido and coworkers ${ }^{42,43}$ is moving towards this direction. Unfortunately, BODIPY dyes are not well suited for single-molecule detection. Attempts were made by Hohsaka and coworkers to introduce larger fluorophores with better photo-physical properties, but they seemed to incorporate only close to the $\mathrm{N}$-terminus of the protein $^{49}$. Since a high flexibility in choosing two eligible positions for smFRET is desirable, our group tried to identify fluorophores with superior characteristics that could be incorporated at desired positions into proteins. We demonstrated the cotranslational incorporation of different dyes, some of them with excellent photo-physical properties suitable for single-molecule measurements. Smaller dyes are in general incorporating more efficiently than large bulky dyes, but the charge of the dyes seem to be even more important. Neutral dyes (e.g. Atto dyes) are better candidates than charged dyes (e.g. Alexa Fluor dyes). For one of them (Atto633) we could show incorporation in combination with a second fluorophore, producing sufficient double-labeled protein to perform smFRET measurements ${ }^{46,50}$. On the way to study cotranslational protein folding, the next step would require the simultaneous incorporation of a pair of fluorophores both well suited for single-molecule measurements.

\section{Development of analytical tools and advanced technical approaches}


Although the use of CFPS potentially can provide valuable samples for smFRET studies, it is still a challenge to obtain enough data for an adequate data analysis that would give interpretable FRET histograms because of the often inherently lower protein yields as compared to the production with cell-based systems. Fortunately, various methodical approaches developed in the last decade improved smFRET detection schemes and provided advanced tools for data evaluation (see for example ref. ${ }^{51}$ ).

Here we discuss tools which are applied for smFRET applications in confocal detection with freely diffusing molecules. Several of the recently developed methods are needed to make use of samples produced by CFPS, in particular to permit a successful analysis of the corresponding data. In this respect we have to take into account that smaller protein yields (with already incorporated dyes or with functional groups for post-translational labeling) do not allow for that degree of protein purification which is typically performed with samples from cell-based approaches. For cell-free produced proteins we generally apply only one purification step employing Ni-NTA magnetic agarose beads for isolating His-tagged labeled target proteins from the CFPS assay ${ }^{27,46,50}$. As a consequence an effective sorting procedure has to be applied to eliminate the contribution of donor-only species to the bursts that finally enter the FRET analysis. Exactly such a treatment is provided by powerful burst analysis tools ${ }^{52}$ and by pulsed interleaved excitation (PIE) ${ }^{53}$ or alternating-laser excitation (ALEX) ${ }^{54,55}$ schemes, which ensure a reasonable quality of the resulting smFRET histograms. However, depending on the total amount of synthesized full length protein and on the dye incorporation or dye attachment efficiency, the number of acceptable burst measured within a given time interval can vary significantly (see Fig. 3). Nevertheless, by employing effective sorting procedures (here PIE) and by using longer data acquisition times we can still achieve reasonable counting statistics to build-up useful smFRET histograms ${ }^{27,50}$.

Another limitation of CFPS-produced proteins is related to the fact that additional experimental measurements (i.e., circular dichroism (CD) spectroscopy, activity assays) of the mutated proteins are often hampered by the small sample amounts. These additional experimental characterizations are typically required to prove the structural and functional integrity of the samples to be used in subsequent smFRET studies. Also in this case we can 
make use of some recent methodical developments that can give reliable information whether the protein shows a correct (native) fold or not.

A

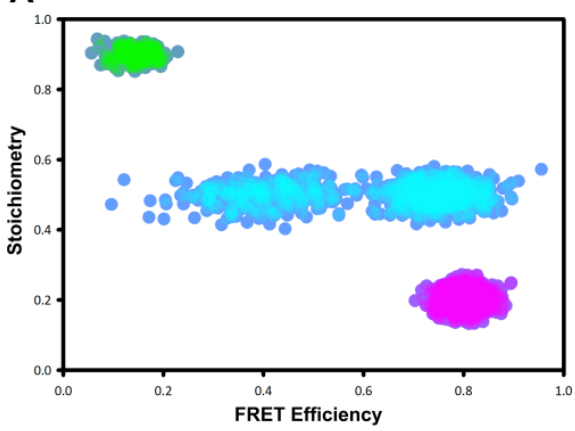

C

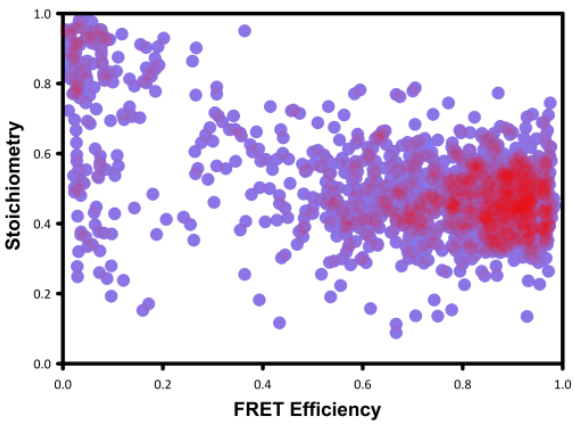

B

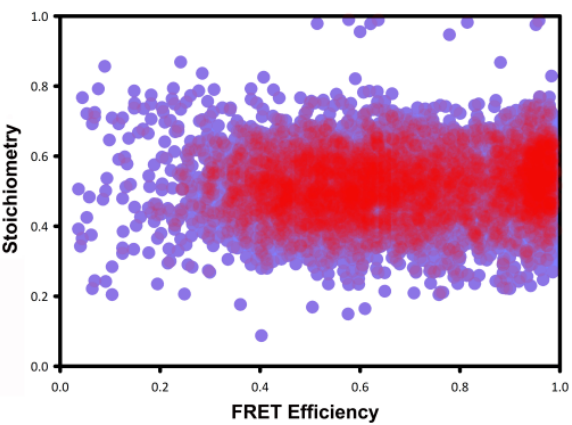

D

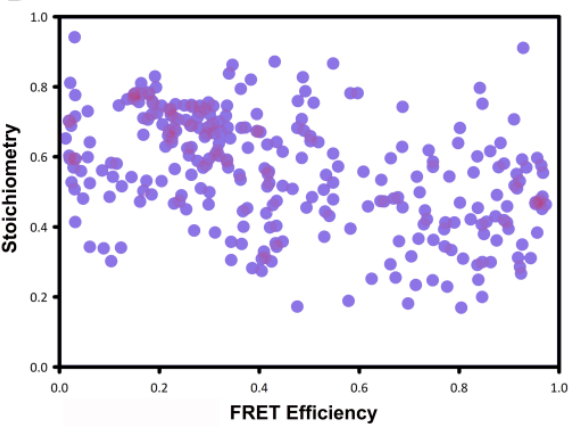

Figure 3. Evaluation of burst statistics in terms of stoichiometry vs. FRET efficiency plots for smFRET data. (A) Simulated data representing the expected behavior for different populations according to Kapanidis et al. ${ }^{54}$ Here donor only populations (green), acceptor only populations (magenta), and two properly labeled populations (high and low FRET with stoichiometry values around 0.5 , in cyan) can be distinguished. In addition plots are shown for real experimental data obtained from calmodulin samples produced with CFPS: (B) post-translationally labeled with Alexa488 (donor) and Alexa647 (acceptor), exhibiting 4,000 accepted bursts per hour, measured for one hour, i.e. 4,000 bursts, (C) posttranslationally labeled with Alexa488 (donor) and co-translationally labeled with Atto633 (acceptor) ${ }^{27}$ exhibiting 400 accepted bursts per hour, measured for three hours, i.e. 1,200 bursts, and (D) cotranslationally labeled with BODIPY-FL (donor) and Atto633 (acceptor) exhibiting 100 accepted bursts per hours, measured for five hours, i.e. 500 bursts. Incorporating UUAs carrying functional groups or fluorescently labeled amino acids reduces the protein yield in CFPS. Although all samples (B-D) were measured with a rather similar protein concentration, which gave approximately 5,000 primary bursts during one hour of measurement, a decreasing number of bursts got accepted (from $B$ to $D$ ) because of a decreasing sample quality (e.g., poor dye quality, high donor-only fraction of full length protein, imperfect sample purification). Figure adapted from ref. ${ }^{50}$. Copyright 2018 American Chemical Society.

First, considering accessible volume (AV) calculation of dyes tethered at a certain position within the protein structure $e^{56-58}$ is a prerequisite to determine the most realistic inter-dye 
distance value, which is later compared with the one obtained from the smFRET measurement. Second, a reasonable agreement between the measured and the modelled inter-dye distances (known from the 3D structure of the investigated protein in combination with the best dye position estimate obtained from $\mathrm{AV}$ calculation) requires the knowledge of the quantum yield values for both dyes, and in particular that of the donor (see ref. ${ }^{59}$ for details). The real values can vary significantly with respect to the often used values obtained from free dyes, mainly due to local quenching. Therefore, the method of choice is to measure the quantum yields of the fluorophores that are attached at these specific positions directly with the SMFRET sample.

We introduced recently a method that allows quantum yield determinations with such small sample amounts ${ }^{60}$. In particular the site-specific in contrast to the residue-specific dye attachment (like in the classical approach with double cysteine mutants) can improve the accuracy of the determined inter-dye distance, as shown recently in a study on calmodulin ${ }^{27}$. Often samples with dyes attached to positions of strong local quenching give rise to significantly broadened FRET histograms and therefore these samples are typically discarded from further consideration. The strength of employing site-specific labeling lies in the fact that this approach intrinsically avoids inaccuracies related to changes in the quantum yield and therefore positions are only discarded if the corresponding quantum yield is too low, which would preclude to get enough photon counts from the dye at that position.

Of course, the benefit of site-specific labeling is not exclusive for samples produced with CFPS, but was introduced already earlier in the case of in vivo protein expression ${ }^{32,61}$. Furthermore, the benefit of potentially more accurate smFRET histograms with site-specifically labeled samples depends strongly on whether pronounced local quenching effects occur. However, by employing the above mentioned methodical approaches we achieved reliable and rather precise inter-dye distance values from smFRET measurements. In this way precise distance values (also for different conformational states if known) provide an informative basis for a correctly folded protein structure. We demonstrated this approach recently in a case study on human calmodulin, also emphasizing the functionality of the cell-free synthesized calmodulin by measuring the interconversion between calcium depleted, calcium bound and peptide bound conformational states ${ }^{27}$. 


\section{Contributions to the field and future perspectives}

The CFPS-based approach to produce proteins for smFRET studies was already performed successfully for various model proteins, calmodulin ${ }^{27}$, barnase ${ }^{25}$, spectrin $^{25}$, T4 lysozyme ${ }^{25}$ and for a glucose binding protein (unpublished results). This indicates that protein sample production with CFPS system has the potential to become a general applicable method. Moreover, a rapid and facile production of a set of samples displaying multiple-distance pairs would also be necessary to manage a more comprehensive protein structure determination ${ }^{62}$. One of the actual trends in smFRET is to investigate distance networks and implement an integrated structural modeling based on FRET data, in order to achieve a better protein structure determination ${ }^{62,63}$. This approach involves the production of many double mutants and requires high accuracy in the distance determination, requirements accomplished in a straight forward manner by the CFPS approach. The introduction of a second orthogonal reactivity for dye attachment in the polypeptide chain, which is not based on the cysteine codon, would also allow larger proteins, typically with many natural cysteines in the sequence, to become applicable for smFRET studies.

In this respect an ideal double labeling approach is to incorporate two UAAs with different bio-orthogonal reactive groups that can be selectively labeled without any cross-reactivity. Most of the approaches however make use of at least one sense codon that has been reassigned to incorporate an $U A A^{27,33,64}$. Thus, smFRET studies are limited to those proteins that have the specific codon quite rarely in their sequence and can tolerate its removal (typically the cysteine). In order to be totally independent of the nascent chain sequence, preferably both UAAs should be incorporated by using two nonsense codons. Attempts in cells using two of the three available stop codons were already met with success ${ }^{29,30}$. Using CFPS instead of cell-based expression would further allow full control of the synthesis of literally any protein with two labels for smFRET. In cells the incorporation of the UAAs using two stop codons is possible because of the rather high protein yield. In CFPS lower yields have to be compensated by depleting the release factors (RFs), which compete with the UAA incorporation at the stop codons. Depletion of RF1 was shown to increase the incorporation efficiency of the UAA ${ }^{65}$ but depletion of both RF1 and RF2 is not possible, since it would have a negative impact on the 
translation of the protein. Thus, the use of two stop codons in CFPS and the incorporation efficiency need to be explored further. Alternatively, different nonsense codons were developed (i.e four- and five-base codons, see Fig. 2$)^{40}$, but the incorporation is even less efficient due to the competition with the corresponding three-base sense codon. Evidently, nonsense codons need to be further optimized, while at the same time more UAAs as well as fluorescent dyes with different reactive groups have to become commercially available. The latter aspect is of high importance for smFRET applicants, because the final goal is to use CFPS for smFRET sample production in a straight-forward manner without any own methodical efforts to produce or optimize the components needed for CFPS.

Another promising approach to push the combination of CFPS and single molecule fluorescence applications forward is given by microfluidic platforms which integrate capillary electrophoresis to obtain a more efficient purification of labeled target proteins. Such devices are ideally suited for very small sample amounts and can easily be combined with singlemolecule fluorescence detection instrumentation ${ }^{66-68}$.

As already mentioned above, the combination of CFPS with smFRET or with other singlemolecule techniques in general unfolds the full potential of studying a complex biological process, like the ribosome catalyzed protein synthesis. In this respect also the polypeptide chain elongation and cotranslational folding of nascent chains would be worthy targets for studies at the single-molecule level ${ }^{19,69}$. The ability to fluorescently label emerging nascent chains is essential in performing studies aiming to understand how protein synthesis and folding are coupled. The flexibility in choosing label positions and the attachment of a dye pair (donor and acceptor) at these positions is of utmost importance for single-molecule fluorescence studies and in particular smFRET.

The most elegant approach for double-labeling a protein in a site-specific and selective way is to cotranslationally incorporate UAAs carrying fluorescent dyes. In a first attempt the cotranslational incorporation of just one single-molecule suitable dye was already demonstrated, but further dye incorporation options are expected in the near future ${ }^{50}$. Here the ultimate goal is to watch a growing nascent chain with cotranslational incorporated dyes by smFRET. Such an approach requires in-situ reactions to be monitored in real time at physiologically relevant 
micro-molar concentration of tRNAs charged with labeled amino acids. Only such rather high concentrations provide a reasonable incorporation yield of fluorescent amino acids into the growing nascent chain. So-called zero-mode waveguides (ZMWs) $)^{70,71}$ provide a technical basis to perform such real time observation of translation and cotranslational folding (Fig. 4). In addition, also the combination of correlated high-resolution force spectroscopy and smFRET measurements ${ }^{6}$ applied to samples from CFPS assays has a high potential to unravel details of co-translational folding.

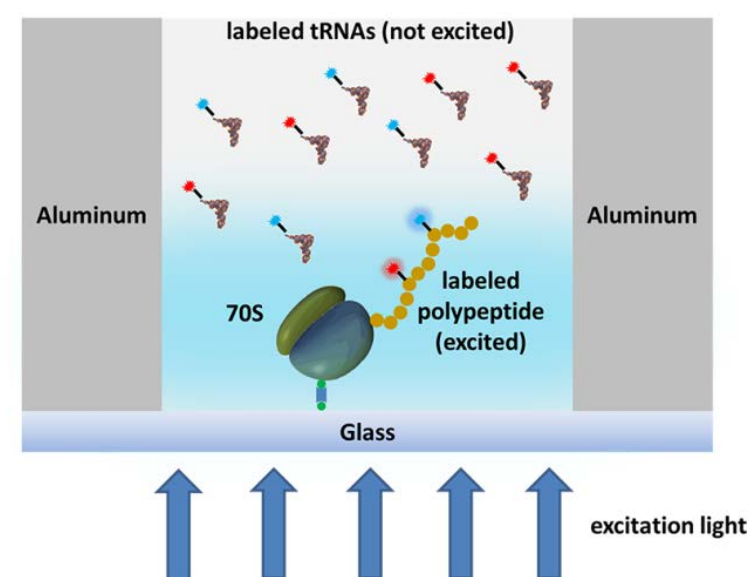

Figure 4. Detection scheme for observing an in situ synthesis reaction monitoring a growing nascent chain by employing ZMWs. Here $70 S$ ribosomes tethered at the bottom of a ZMW are synthesizing a nascent polypeptide chain. By employing a commercial CFPS kit including exogenous tRNAs, charged with labeled amino acids, a site selective incorporation of a donor/acceptor pair into the growing nascent chain is accomplished. The width of the ZMW is approximately $50 \mathrm{~nm}$ and the limited penetration depth of the excitation light is roughly $25 \mathrm{~nm}$ (blue shaded area). In principle such a detection scheme should allow to obtain sMFRET data from individual nascent chain, since the excess of labeled tRNAs is mainly present in the area which is not excited. If nevertheless these tRNAs diffuse into the excitation volume they will either bind to the ribosome and subsequently be incorporated into the nascent chain or will diffuse out of this area within rather short time intervals. Therefore, a FRET signal which remains for a certain time interval will give valuable information about the structural properties (e.g., folding states) of the growing nascent chain.

In summary, we can state that CFPS, mainly by employing commercially available purified and reconstituted in vitro systems to better control translation and thus incorporation of UAAs, has the potential to become a more relevant method in future to produce protein samples for smFRET studies. The advantages are that (i) CFPS is fast and allows for a rapid screening of 
feasible mutants, (ii) the incorporation of unnatural amino acids, even though it falls short compared to the efficiency and specificity of natural protein synthesis and needs to be further optimized, it still provides a site specific, and most importantly a site selective label scheme, which is the basis for the highest accuracy of measured data, and (iii) CFPS extends the scope of application to toxic or difficult to express proteins. In this respect CFPS and smFRET represent a perfect combination to achieve the full potential of analyzing protein structures with fluorescence based techniques.

\section{Acknowledgements}

We would like to express our gratitude to our former co-workers Dr. M. Sadoine, Dr. M. Gerrits, and Dr. M. Cerminara who contributed substantially to the results discussed in this publication.

\section{ORCID}

Alexandros Katranidis: 0000-0002-1785-1659

Jörg Fitter: 0000-0002-4503-2079

\section{References}

(1) Michalet, X.; Weiss, S.; Jager, M. Chem. Rev. 2006, 106, 1785-1813.

(2) Joo, C.; Balci, H.; Ishitsuka, Y.; Buranachai, C.; Ha, T. Annu. Rev. Biochem. 2008, 77, 51-76.

(3) Schuler, B.; Eaton, W. A. Curr. Opin. Struct. Biol. 2008, 18, 16-26.

(4) Fitter, J.; Katranidis, A.; Rosenkranz, T.; Atta, D.; Schlesinger, R.; Büldt, G. Soft Matter 2011, 7, 12541259.

(5) Sustarsic, M.; Kapanidis, A. N. Curr. Opin. Struct. Biol. 2015, 34, 52-59.

(6) Hashemi Shabestari, M.; Meijering, A. E. C.; Roos, W. H.; Wuite, G. J. L.; Peterman, E. J. G. Methods Enzymol. 2017, 582, 85-119.

(7) Sharma, S.; Chakraborty, K.; Muller, B. K.; Astola, N.; Tang, Y. C.; Lamb, D. C.; Hayer-Hartl, M.; Hartl, F. U. Cell 2008, 133, 142-153.

(8) Pirchi, M.; Ziv, G.; Riven, I.; Cohen, S. S.; Zohar, N.; Barak, Y.; Haran, G. Nat. Commun. 2011, 2, 493.

(9) Shimizu, Y.; Inoue, A.; Tomari, Y.; Suzuki, T.; Yokogawa, T.; Nishikawa, K.; Ueda, T. Nat. Biotechnol. 2001, 19, 751-755.

(10) Katzen, F.; Chang, G.; Kudlicki, W. Trends Biotechnol. 2005, 23, 150-156.

(11) Ohashi, H.; Kanamori, T.; Shimizu, Y.; Ueda, T. Curr. Pharm. Biotechnol. 2010, 11, 267-271.

(12) Carlson, E. D.; Gan, R.; Hodgman, C. E.; Jewett, M. C. Biotechnol. Adv. 2012, 30, 1185-1194.

(13) Rosenblum, G.; Cooperman, B. S. FEBS Lett. 2014, 588, 261-268. 
(14) Uemura, S.; lizuka, R.; Ueno, T.; Shimizu, Y.; Taguchi, H.; Ueda, T.; Puglisi, J. D.; Funatsu, T. Nucleic Acids Res. 2008, 36, e70.

(15) Katranidis, A.; Atta, D.; Schlesinger, R.; Nierhaus, K. H.; Choli-Papadopoulou, T.; Gregor, I.; Gerrits, M.; Büldt, G.; Fitter, J. Angew. Chem. Int. Ed. Engl. 2009, 48, 1758-1761.

(16) Zhou, Z. P.; Shimizu, Y.; Tadakuma, H.; Taguchi, H.; Ito, K.; Ueda, T. J. Biochem. 2011, 149, 609-618.

(17) Saraogi, I.; Zhang, D.; Chandrasekaran, S.; Shan, S. O. J. Am. Chem. Soc. 2011, 133, 14936-14939.

(18) Kaiser, C. M.; Goldman, D. H.; Chodera, J. D.; Tinoco, I., Jr.; Bustamante, C. Science 2011, 334, 17231727.

(19) Wruck, F.; Katranidis, A.; Nierhaus, K. H.; Büldt, G.; Hegner, M. Proc. Natl. Acad. Sci. USA 2017, 114, E4399-E4407.

(20) Blanchard, S. C.; Gonzalez, R. L.; Kim, H. D.; Chu, S.; Puglisi, J. D. Nat. Struct. Mol. Biol. 2004, 11, 1008-1014.

(21) Marshall, R. A.; Aitken, C. E.; Dorywalska, M.; Puglisi, J. D. Annu. Rev. Biochem. 2008, 77, 177-203.

(22) Perez, C. E.; Gonzalez, R. L., Jr. Curr. Opin. Chem. Biol. 2011, 15, 853-863.

(23) Ohashi, H.; Shimizu, Y.; Ying, B. W.; Ueda, T. Biochem. Biophys. Res. Commun. 2007, 352, 270-276.

(24) Ha, T. Nat. Methods 2014, 11, 1015-1018.

(25) Hamadani, K. M.; Howe, J.; Jensen, M. K.; Wu, P.; Cate, J. H. D.; Marqusee, S. J. Biol. Chem. 2017, 292, 15636-15648.

(26) Kempf, N.; Remes, C.; Ledesch, R.; Züchner, T.; Höfig, H.; Ritter, I.; Katranidis, A.; Fitter, J. Sci. Rep. 2017, 7, 46753.

(27) Sadoine, M.; Cerminara, M.; Kempf, N.; Gerrits, M.; Fitter, J.; Katranidis, A. Anal. Chem. 2017, 89, 11278-11285.

(28) Wang, L.; Brock, A.; Herberich, B.; Schultz, P. G. Science 2001, 292, 498-500.

(29) Wu, B.; Wang, Z.; Huang, Y.; Liu, W. R. ChemBioChem 2012, 13, 1405-1408.

(30) Kim, J.; Seo, M. H.; Lee, S.; Cho, K.; Yang, A.; Woo, K.; Kim, H. S.; Park, H. S. Anal. Chem. 2013, 85, 1468-1474.

(31) Wang, L.; Xie, J.; Schultz, P. G. Annu. Rev. Biophys. Biomol. Struct. 2006, 35, 225-249.

(32) Brustad, E. M.; Lemke, E. A.; Schultz, P. G.; Deniz, A. A. J. Am. Chem. Soc. 2008, 130, 17664-17665.

(33) Seo, M. H.; Lee, T. S.; Kim, E.; Cho, Y. L.; Park, H. S.; Yoon, T. Y.; Kim, H. S. Anal. Chem. 2011, 83, 8849-8854.

(34) Soriano Del Amo, D.; Wang, W.; Jiang, H.; Besanceney, C.; Yan, A. C.; Levy, M.; Liu, Y.; Marlow, F. L.; Wu, P. J. Am. Chem. Soc. 2010, 132, 16893-16899.

(35) Uttamapinant, C.; Tangpeerachaikul, A.; Grecian, S.; Clarke, S.; Singh, U.; Slade, P.; Gee, K. R.; Ting, A. Y. Angew. Chem. Int. Ed. Engl. 2012, 51, 5852-5856.

(36) Jewett, J. C.; Bertozzi, C. R. Chem. Soc. Rev. 2010, 39, 1272-1279.

(37) Plass, T.; Milles, S.; Koehler, C.; Schultz, C.; Lemke, E. A. Angew. Chem. Int. Ed. Engl. 2011, 50, 38783881.

(38) Hohsaka, T.; Ashizuka, Y.; Sasaki, H.; Murakami, H.; Sisido, M. J. Am. Chem. Soc. 1999, 121, 1219412195.

(39) Hohsaka, T.; Ashizuka, Y.; Taira, H.; Murakami, H.; Sisido, M. Biochemistry 2001, 40, 11060-11064.

(40) Hohsaka, T.; Sisido, M. Curr. Opin. Chem. Biol. 2002, 6, 809-815.

(41) Quast, R. B.; Mrusek, D.; Hoffmeister, C.; Sonnabend, A.; Kubick, S. FEBS Lett. 2015, 589, 1703-1712.

(42) Kajihara, D.; Abe, R.; lijima, I.; Komiyama, C.; Sisido, M.; Hohsaka, T. Nat. Methods 2006, 3, 923-929.

(43) lijima, I.; Hohsaka, T. ChemBioChem 2009, 10, 999-1006.

(44) Perronet, K.; Bouyer, P.; Westbrook, N.; Soler, N.; Fourmy, D.; Yoshizawa, S. J. Lumin. 2007, 127, 264-268.

(45) Dulin, D.; Le Gall, A.; Perronet, K.; Soler, N.; Fourmy, D.; Yoshizawa, S.; Bouyer, P.; Westbrook, N. Phys. Procedia 2010, 3, 1563-1567. 
(46) Sadoine, M.; Cerminara, M.; Fitter, J.; Katranidis, A. Bio Protoc. 2018, 8, e2881.

(47) Gershenson, A.; Gierasch, L. M. Curr. Opin. Struct. Biol. 2011, 21, 32-41.

(48) Thommen, M.; Holtkamp, W.; Rodnina, M. V. Curr. Opin. Struct. Biol. 2017, 42, 83-89.

(49) Abe, R.; Shiraga, K.; Ebisu, S.; Takagi, H.; Hohsaka, T. J. Biosci. Bioeng. 2010, 110, 32-38.

(50) Sadoine, M.; Cerminara, M.; Gerrits, M.; Fitter, J.; Katranidis, A. ACS Synth. Biol. 2018, 7, 405-411.

(51) Hellenkamp, B.; Schmid, S.; Doroshenko, O.; Opanasyuk, O.; Kuhnemuth, R.; Rezaei Adariani, S.; Ambrose, B.; Aznauryan, M.; Barth, A.; Birkedal, V.; Bowen, M. E.; Chen, H.; Cordes, T.; Eilert, T.; Fijen, C.; Gebhardt, C.; Gotz, M.; Gouridis, G.; Gratton, E.; Ha, T.; Hao, P.; Hanke, C. A.; Hartmann, A.; Hendrix, J.; Hildebrandt, L. L.; Hirschfeld, V.; Hohlbein, J.; Hua, B.; Hubner, C. G.; Kallis, E.; Kapanidis, A. N.; Kim, J. Y.; Krainer, G.; Lamb, D. C.; Lee, N. K.; Lemke, E. A.; Levesque, B.; Levitus, M.; McCann, J. J.; NarediRainer, N.; Nettels, D.; Ngo, T.; Qiu, R.; Robb, N. C.; Rocker, C.; Sanabria, H.; Schlierf, M.; Schroder, T.; Schuler, B.; Seidel, H.; Streit, L.; Thurn, J.; Tinnefeld, P.; Tyagi, S.; Vandenberk, N.; Vera, A. M.; Weninger, K. R.; Wunsch, B.; Yanez-Orozco, I. S.; Michaelis, J.; Seidel, C. A. M.; Craggs, T. D.; Hugel, T. Nat. Methods 2018, 15, 669-676.

(52) Nir, E.; Michalet, X.; Hamadani, K. M.; Laurence, T. A.; Neuhauser, D.; Kovchegov, Y.; Weiss, S. J. Phys. Chem. B 2006, 110, 22103-22124.

(53) Müller, B. K.; Zaychikov, E.; Bräuchle, C.; Lamb, D. C. Biophys. J. 2005, 89, 3508-3522.

(54) Kapanidis, A. N.; Lee, N. K.; Laurence, T. A.; Doose, S.; Margeat, E.; Weiss, S. Proc. Natl. Acad. Sci. USA 2004, 101, 8936-8941.

(55) Kudryavtsev, V.; Sikor, M.; Kalinin, S.; Mokranjac, D.; Seidel, C. A. M.; Lamb, D. C. ChemPhysChem 2012, 13, 1060-1078.

(56) Muschielok, A.; Andrecka, J.; Jawhari, A.; Brückner, F.; Cramer, P.; Michaelis, J. Nat. Methods 2008, 5, 965-971.

(57) Sindbert, S.; Kalinin, S.; Nguyen, H.; Kienzler, A.; Clima, L.; Bannwarth, W.; Appel, B.; Müller, S.; Seidel, C. A. J. Am. Chem. Soc. 2011, 133, 2463-2480.

(58) Höfig, H.; Gabba, M.; Poblete, S.; Kempe, D.; Fitter, J. Molecules 2014, 19, 19269-19291.

(59) Kempe, D.; Cerminara, M.; Poblete, S.; Schöne, A.; Gabba, M.; Fitter, J. Anal. Chem. 2017, 89, 694702.

(60) Kempe, D.; Schone, A.; Fitter, J.; Gabba, M. J. Phys. Chem. B 2015, 119, 4668-4672.

(61) Lemke, E. A. Methods Mol. Biol. 2011, 751, 3-15.

(62) Dimura, M.; Peulen, T. O.; Hanke, C. A.; Prakash, A.; Gohlke, H.; Seidel, C. A. Curr. Opin. Struct. Biol. 2016, 40, 163-185.

(63) Sali, A.; Berman, H. M.; Schwede, T.; Trewhella, J.; Kleywegt, G.; Burley, S. K.; Markley, J.; Nakamura, H.; Adams, P.; Bonvin, A. M.; Chiu, W.; Peraro, M. D.; Di Maio, F.; Ferrin, T. E.; Grünewald, K.; Gutmanas, A.; Henderson, R.; Hummer, G.; Iwasaki, K.; Johnson, G.; Lawson, C. L.; Meiler, J.; Marti-Renom, M. A.; Montelione, G. T.; Nilges, M.; Nussinov, R.; Patwardhan, A.; Rappsilber, J.; Read, R. J.; Saibil, H.; Schröder, G. F.; Schwieters, C. D.; Seidel, C. A.; Svergun, D.; Topf, M.; Ulrich, E. L.; Velankar, S.; Westbrook, J. D. Structure 2015, 23, 1156-1167.

(64) Cui, Z.; Mureev, S.; Polinkovsky, M. E.; Tnimov, Z.; Guo, Z.; Durek, T.; Jones, A.; Alexandrov, K. ACS Synth. Biol. 2017, 6, 535-544.

(65) Gerrits, M.; Strey, J.; Claußnitzer, I.; von Groll, U.; Schäfer, F.; Rimmele, M.; Stiege, W. In Cell-free Protein Expression, Kudlicki, W. A.; Katzen, F.; Bennett, R. P., Eds.; Landes Bioscience: Austin, Tex., 2007, pp 166-180.

(66) Schiro, P. G.; Kuyper, C. L.; Chiu, D. T. Electrophoresis 2007, 28, 2430-2438.

(67) Kim, S.; Huang, B.; Zare, R. N. Lab Chip 2007, 7, 1663-1665.

(68) Wang, C.; Ouyang, J.; Ye, D. K.; Xu, J. J.; Chen, H. Y.; Xia, X. H. Lab Chip 2012, 12, 2664-2671.

(69) Holtkamp, W.; Kokic, G.; Jäger, M.; Mittelstaet, J.; Komar, A. A.; Rodnina, M. V. Science 2015, 350, 1104-1107. 
(70) Levene, M. J.; Korlach, J.; Turner, S. W.; Foquet, M.; Craighead, H. G.; Webb, W. W. Science 2003, 299, 682-686.

(71) Zhu, P.; Craighead, H. G. Annu. Rev. Biophys. 2012, 41, 269-293. 


\section{FOR TABLE OF CONTENTS ONLY}

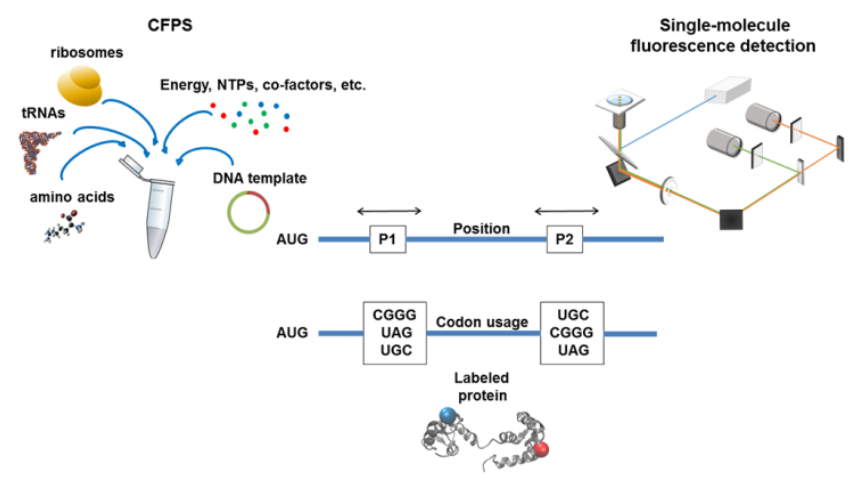

